\title{
EFFECT OF MAGNETIC ATTACHMENT FLUX ON SUB-GINGIVAL PIGMENTED BACTEROIDES MELANINOGENICUS COUNT IN MAGNETICALLY RETAINED OVERDENTURE WEARERS
}

\author{
Khaled K. El-Din Amin* and Sahar MN Bukhary **
}

\begin{abstract}
Statement of problem: Dental magnetic attachments has electromagnetic flux that could affect bacterial certain species growth, how would be its effect on a subgingival bacterial specie that had been isolated from both healthy and periodontally affected gingival crevice.

Aim of the study is recognition the effect of using dental magnetic attachment on subgingival Black pigmented Bacteroides.

Material and Method Ten male patients were selected and received magnetically retained overdenture on mandibular canines. Subgingival samples were collected and were serially diluted. $0.1 \mathrm{ml}$ was transferred from each dilution on the surface of blood agar plates which incubated anaerobically. Black pigmented colonies of bacteroides melaninogenicus were counted and the colony forming units per sample (CFU) was obtained.
\end{abstract}

Results and Conclusion significant decrease in bacteroides counts were observed after one week and a month of magnet fixation. Magnetic dental attachment flux could have an inhibitory effect on subgingival pigmented bacteroides melaninogenicus.

KEY WORDS Dental Magnetic flux-Magnetically retained overdenture-Subgingival black pigmented bacteroides.

\section{INTRODUCTION}

Thomas, $1995^{(36)}$ reported that magnetic attachments were superior in comparison with barclip retention because it did not provide stresses, it is considered as a break up element to lateral forces and there was no need of abutment parallism. Moreover, Bhat et al., $2013^{(6)}$ and Raghavan et al., $2015^{(28)}$ concluded that dental magnetic attachment has a benefit of no special fixation techniques is required. It also could be used with 24 degree

*Associate Professor, Oral and Maxillofacial Prosthodontics Department, Faculty of Dentistry, King Abdulaziz University ** Associate Professor, Department of Oral Biology, Faculty of Dentistry, King Abdulaziz University, 
divergent abutments and have an easier path of insertion of prosthesis if compared with other interlocking attachments.

Ceruti et al $201{ }^{(9)}$ also added that magnetic attachments came superior to other mechanical attachments as ball or bar forms in retention quality of prosthetic appliance. Chu et al 2004 ${ }^{(10)}$ stated that the magnetic-retained dentures were easier in use for patients with physical disabilities.

Jena et al., 2003 ${ }^{(13)}$ used two combined magnetically retained maxillofacial prosthesis and could overcome problem of path of insertion in such cases. While Lemon et al, $1996^{(18)}$ could use a technique involved samarium cobalt magnets embedded in silicone prosthesis and counter magnets in a holding device to restore a lateral nasal defect. On the

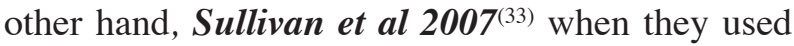
open field rare -earth magnets to retain artificial eye, occurrence of localized exophytic bone formation around exenterated orbit was a reported.

According to type of magnetic field, Bhat et al. $2013{ }^{(6)}$ classified the studied different dental magnets into open and closed magnetic field. They added that samarium cobalt magnets like Dyna magnetic attachment had high magnetization capacity and stable with high thermal application.

Raghavan et al., $2015^{(28)}$ consider Dyna_magnet as an open field magnetic attachment and mentioned that magnetic flux density refers to strength of magnetic field and measured by millitesla. Moreover, Portnoy, $1986^{(27)}$ reported that there was no absolute closed magnetic system. The closure was a variable

On an animal study, Tenford, $1994^{(35)}$ concluded that static magnetic field with flux density of 2 tesla hadn't abnormal behavioral and/or physiological alterations. The leakage of magnetic field of different dental magnetic attachments in Hosoi et $\boldsymbol{a l}, \mathbf{2 0 0 8}^{(12)}$ study was limited $5 \mathrm{~mm}$ away from magnetic attachment. Authors confirmed that flux strength should not be more than 40 millitesla as
WHO specks permit. Jena et al., $2003^{(13)}$ reviewed that static magnetic field of Sm-Co magnets could affect fibroblast activity. Moreover orthodontic magnetic brackets of 130 gauss had an effect on microbial flora in mouth. While Kameda and Ohkuma $2014^{(14)}$ found that bacteria could corrode stainless steel of orthodontic appliances while magnetic field has a protective effect against this type of corrosion.

The plaque and bacteria are not different things because plaque always grow due to microbial colonization stared by bacterial adhesion to salivary glycoprotein, then division and accumulation or colonization of bacteria with other types lead to formation of mature plaque as suggested by Mcghee et al. $1982^{(20)}$. They found that increased plaque with its bacterial contents were associated with gingival inflammation.

Savitt and Socransky $1984^{(30)}$ noted that black pigmented bacteroides were isolated in $20 \%$ in samples of healthy sites, $42 \%$ of gingivitis samples, $61 \%$ of adult periodontitis and $73 \%$ in juvenile periodontitis cases. They added that the plaque sample may be a reflection of prior attachment loss, i-e the sample may be a reflection of prior phase of periodontal disease.

Moreover, Newman et al $1976^{(22)}$ concluded the same result mentioned about the pathogenicity of Gram- ve anaerobic rods in animals which suggesting their diagnostic role in cases of periodontosis.

Tanner et al, $1984^{(37)}$ correlated the radiographs taken every three months with dominant microflora and they found with recent bone loss, high percentage of bacteroides gingival is, fusiform cells and small amounts of spirochetes were detected.

\section{MATERIAL AND METHOD}

Ten nonsmoker male patients were selected from the out-patient clinic of the prosthodontics department, Faculty of Oral and Dental Medicine, Nahda University. Their ages ranged between $42-$ 64 years old. 
Selection was performed according to the following Criteria:

1- Free from any systemic disease as indicated by medical history and clinical inspection.

2- Having mandibular canines bilaterally supported by sufficient alveolar bones and does not show inflammatory signs of gum and periodontal pockets were $\leq 3 \mathrm{~mm}$. (Aas et al 2005) ${ }^{(1)}$

3- Mandibular canines' abutments were free from Periapical radiolucency as indicated by Periapical X-ray films.

4- Tooth mobility, if present, was not more than grade I mobility.

5- Healthy firm mucosa covering the edentulous ridge.

6- Patients were not smoker (Könönen \& Kumar 2015) ${ }^{(16)}$

7- Cooperative patients and could be motivated for instructions for oral hygiene.

8- Written informed consent was obtained from participants who agreed to participate voluntarily. The study protocol was approved by the Ethical Committee of NAHDA University.

The mandibular canines that will serve as abutments for the overdenture were kept and all other hopeless remaining teeth were extracted. The patients were left for a period of 10-12 weeks after extraction to allow tissue healing and first bone remoulding. Root canal therapy was performed for the abutments using gutta percha points for final obliteration.

- The clinical crown was cut down about $1 \mathrm{~mm}$ above the free gingival margin to create enough space for the magnets inside the denture base.

- An intra - radicular conical preparation of a depth of approximately $5 \mathrm{~mm}$ was made within each abutment.

- Upper conventional complete denture was constructed for each patient. Mandibular overden- ture was constructed on master cast produced from monophase rubber base impression material while magnetic keeper was temporarily cemented intra-radiculary.

- Before separation of the denture from the cast, laboratory remounting was made. The occlusion was refined again at the time of insertion and pressure indicating paste was used to ensure no pressure areas of delivered dentures upon bearing soft tissues and free gingivae of abutments.

- The patients were asked to come back shortly to correct any complaint after the denture insertion and motivated for cleaning fitting surface of denture under running water after meals while abutments to be cleaned gently with a new moist soft dental brush three times daily without using tooth pastes (Haffajee et al $2001^{(11)}$ and Brkovic et al $\left.2015^{(8)}\right)$. Patients were also instructed to avoid antibiotic medication/or mouth wash one week before bacteriologic samples collection in follow up periods (Williams. 1963 ${ }^{(39)}$ and Walker et al 1981) ${ }^{(38)}$ and inform authors if this happened.

- After two weeks of overdenture insertion and ensuring patients were using dentures comfortably and before seating the magnets assembly, sub gingival samples were collected for each patient (Referred as control A) and subgingival samples were taken after one week (referred as $\mathbf{B}$ ) and one month of magnet fixation (referred as $\mathbf{C}$ )

\section{Bacteriological study}

- Specimens: Were obtained gently and deep from the labial gingival crevice of both abutments (Könönen and Kumar $2015^{(16)}$ and Omar A.A. et al 1990) ${ }^{(25)}$ using a sterile periodontal curette (Aas et al 2005) ${ }^{(25)}$.

- After removal of supragingival plaque, the first curettage samples were collected (Mombelli et al 1988) ${ }^{(21)}$ from bottom of gingival crevice, while abutments were isolated (Hafjee et al $2001^{(11)}$ \& Shchipkova et al 2010) ${ }^{(31)}$. 


\section{Bacterial Count of B. Melaninogenicus:}

1- Every patient samples were carefully homogenized without aeration into $10 \mathrm{ml}$ of Schaedler's broth to make 1 in 10 , dilution.

2- Using a sterile pipette, $1 \mathrm{ml}$ of the previous dilution was added to $10 \mathrm{ml}$ of broth to obtain a dilution of 1 in 100. This step was repeated to obtain a third dilution of 1 in 1000 by a sterile micropipette.

3- $0.1 \mathrm{ml}$ was transferred from each dilution on the surface of blood agar plates supplement with kanamycin-vancomycine.

4- Plates were incubated anaerobically, as they placed in the anaerobic jar where gas generating pack was opened and $10 \mathrm{ml}$ distilled water added., an indicator strip was placed in the clamp under the lid, then the jar was tightly closed and put in the incubator at $37 \mathrm{c}$ for 72 hours.

5- After incubation, the tintorial characterized black pigmented colonies of bacteroides melaninogenicus were counted in every plate and multiplied by the corresponding dilution factor and by ten to obtain the colony forming units per sample (CFU).
The intra-oral magnet of 500gm magnetic power was seated intra- orally by the Direct Technique (Dyna Manual, 1993) (Dyna Dental Engineering B.V. Halsteren, The Netherlands) as following

At the delivery appointment of magnetically retained overdentures, patients were remotivated reviewed for good oral hygiene measures and instructed to avoid any destructive procedures for the magnets by instructing them as follows.

- Clean dentures after meals using tap water.

- Brushing the abutments using moist soft brush.

- Avoiding the metal scrubbing for the magnets.

- Coming back to correct any complaints if present.

After one week and one month of magnets fixation while patients regularly use magnetically retained overdenture (Akin and Ozdemir2013) ${ }^{(3)}$, subgingival specimens were taken in the same manner as before magnet fixation. Data of results for each patient at different follow up periods were collected, tabulated and statistically analyzed Student's ' $t$ 'test was used to compare the data and the $\mathrm{p}$ value was calculated using SPSS statistical program (Inc., Chicago, Illinois, USA.). Statistical significance was determined at a level of $\boldsymbol{P}$ less than 0.05 .

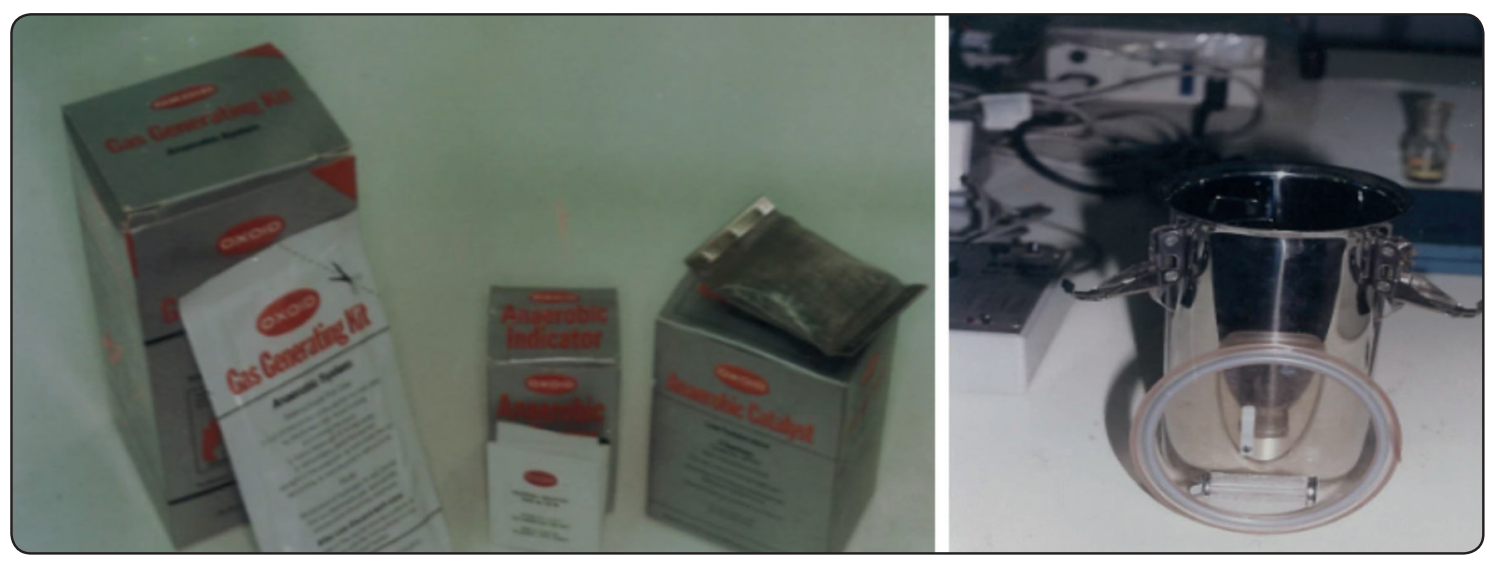

Fig. (1) An aerobic kit and anaerobic jar 

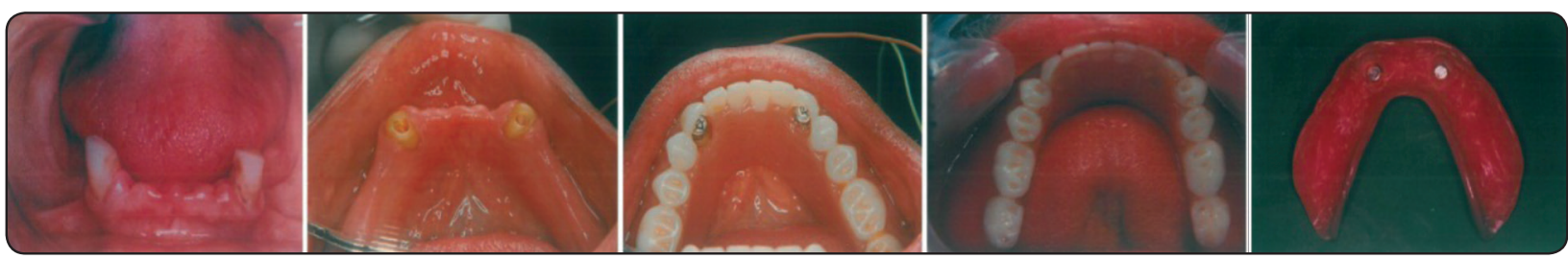

Fig. (2) Abutment preparation and Magnet fixation to mandibular overdenture

\section{RESULTS}

TABLE (1) Mean and standard deviation of bacteroides melaninogenicus at different follow up periods:

\begin{tabular}{|l|c|c|}
\hline \multicolumn{1}{|c|}{ Follow up periods } & Mean & Std. Deviation \\
\hline Before magnet fixation (A) & 4.22 & 1.653817 \\
\hline One week after magnet fixation (B) & 2.88 & 1.189584 \\
\hline One month after magnet fixation (C) & 2.96 & 1.248288 \\
\hline
\end{tabular}

TABLE (2) Comparison of mean bacteroides Melaninogenicus count between different follow up periods and the significance:

\begin{tabular}{|l|c|c|}
\hline \multicolumn{1}{|c|}{$\begin{array}{c}\text { Comparison between } \\
\text { follow up periods }\end{array}$} & $\begin{array}{c}\text { Mean } \\
\text { difference }\end{array}$ & Significance \\
\hline A and B & 1.34 & 0.039 \\
\hline A and C & 1.26 & 0.051 \\
\hline B and C & -.08 & 0.898 \\
\hline
\end{tabular}

*. The mean difference is significant at the 0.05 level.

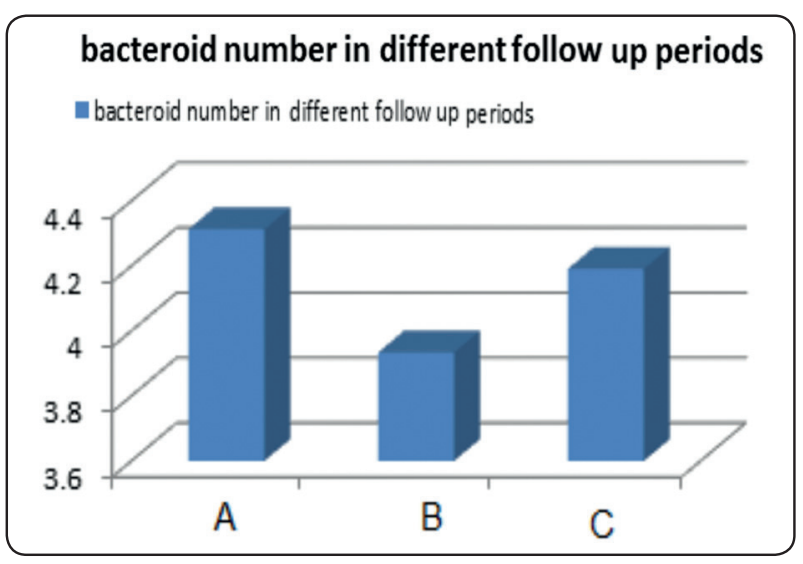

Fig. (3)
After one week of magnet fixation (B), mean of bacteroides count was reduced and showed significant difference when statistically compared with that of samples taken before magnet fixation (A). The mean difference between samples collected after one month and those collected before magnet fixation was also significant. Plaque samples of seven patients out of the ten in one month follow up (C) showed increasing than corresponding counts of the same patients of one week follow up (B) and the mean difference between the two periods was insignificant.

\section{DISCUSSION}

The patients were selected with minimal pocket depth as failure of overdenture were almost attributed to inadequate periodontal condition as reported by Aas et al $2005^{(1)}$ \& Robbins $1980^{(29)}$. Patients were nonsmoker as smoking could affect level of chemokines, cytokines and growth factors in gingival crevice which subsequently cause shifting in the subgingival microbiome as mentioned by Könönen and Kumar 2015 ${ }^{(16)}$.

The female patients were excluded from the study as Kumar 2013 ${ }^{(15)}$ and Nirola A et al., 2018 24) $^{(24)}$ reported fluctuating female sex hormones has an effect on gingival crevicular fluids and subgingival microflora shifting

The patients were instructed to use moist brush without any dentifrices in cleaning abutments to eliminate an expected effect of dentifrices on oral microflora as reported by Haffajee AD et al 2001 ${ }^{(11)}$ and Brkovic et al $2015^{(8)}$. 
Patients were also motivated to avoid usage of antibiotics minimum one week before bacterial samples collection as this could affect the crevicular bacterial flora Walker et al $1981^{(38)}$.

Plaque samples were collected gently deep from gingival crevice to standardize subgingival sampling technique as reported by (Könönen and Kumar $2015^{(16)}$ and furthermore supragingival plaque was removed before subgingival sampling and abutments were isolated to avoid contamination of saliva upon collected samples as stated by Shchipkova et al $2010^{(31)}$.

Using monoplane occlusion in setting up the overdenture teeth to eliminate horizontal forces that could subsequently affect the periodontal health state.

Follow up period were formulated not to exceed one month after magnet fixation to avoid an expected effect of corrosive surrounding media on dental magnets as concluded by Chung $\boldsymbol{C ~ H}$, et al $2005^{(10)}$ Moreover, Boeckler et al. $2009^{(8)}$ stated according to ISO 10271 that modern magnetic attachment showed metal ions dissolved in all corrosive medium specimen within few weeks of use.

The significant decrease of subgingival bacteroides count after one week of magnet fixation could be attributed to inhibitory effect of static magnetic flux on bacterial flora growth as concluded by Kohno et al 2000 ${ }^{(17)}$. Moreover, Abdelkader et al $2012^{(2)}$ and Bajpa et al 201 ${ }^{(5)}$ reported same effect of magnetic field on the studied pathogenic microbes.

There was also significant mean reduction in bacteroides count after one month of magnet fixation. While the mean difference between one month and one week bacteroides counts was nonsignificant without pocket depth variation. This could be explained by starting of inflammatory condition which start shifting of subgingival microflora especially anaerobic gram negative organisms in periodontally affected abutments as stated by Savitt and Socransky $1984^{(37)}$, Maiden et al, $1990^{(19)}$, who reported $45 \%$ of the total isolates and White and D. Mayrand 1981 ${ }^{(37)}$ who specified that $31.8 \%$ of isolated organisms were bacteroides sachrolyticcus. Moreover, Parekh M, et al 2016 ${ }^{(26)}$ reported 69 isolates out of 89 were gram negative anaerobes in patients with chronic periodontitis with an average age of 54 years. On other side, Morhart RE, Fitzgerald RJ 1976 ${ }^{(23)}$ and Ali $S$ M F, and Tanwir F 2018(4) concluded that oral microflora represents a dynamic entity and its shift could be also according to food metabolites and change of ph.

\section{CONCLUSION}

Under the limitation of this study. Magnetic dental attachment flux has an inhibitory effect on subgingival black pigmented bacteroides count.

\section{Recommendation:}

- In vivo studies could be continued upon effect of dental magnetic flux on subgingival microflora accompanied by early specific gingival inflammatory mediators and late clinical signs for more extended time of follow up periods.

- In vitro studies could be continued to study the effect of magnetic flux on certain species in oral microbial flora with respect to ecosystem of subgingival microflora, $\mathrm{Ph}$ and certain food intake effect specially carbohydrate.

\section{REFERENCES}

1. Aas J A, Paster BJ, Stokes L N, Olsen I, and Dewhirst F E, Defining the Normal Bacterial Flora of the Oral Cavity, Journal Of Clinical Microbiology. 2005, 43(11):57215732 .

2. Abdel -Kader H M, Aref MI, and Yousef SW: The biological effects of static magnetic field of commercial samarium -Cobalt (SmCo5) orthodontic magnets on cultured Escherichia coli and staphylococci aureus .Int $\mathrm{J}$ Clin Dent. 2012; 5(3):201-10.

3. Akin $\mathrm{H}$ and Ozdemir A K: Effect of corrosive environments and thermocycling on the attractive force of four types of 
dental magnetic attachments, Journal of Dental Sciences .2013, 8: 184-188.

4. Ali S M F, and Tanwir F;Oral microbial habitat a dynamic entity journal of Oral Biology and Craniofacial Research 2012 September-December Volume 2, Number 3; pp. $181 \mathrm{e} 187$

5. Bajpa I, Saha N, and Basu B: Moderate intensity static magnetic field has bactericidal effect on E. coli and S.epidermidis on sintered hydroxyapatite. J Biomed Mater Res B Appl Biomater.2012; 100(5):1026-17.

6. Bhat V S, Shenoy KK, Premkumar P. Magnets in dentistry, Archives of Medicine and Health Sciences, Jan-Jun 2013, 1( 1): 73-79

7. Boeckler AF, Ehring C, Morton D, Geis-Gerstorfer J, Setz JM Corrosion of Dental Magnet Attachments for Removable Prostheses on Teeth and Implants, Journal of Prosthodontics. 2009, 18, 301-308.

8. Brkovic S, Postic S, and Ilic D: Influence of the magnetic field on microorganisms in the oral cavity Appl Oral Sci., 2015, 23(2):179-86.

9. Ceruti P, Bryant SR, Lee JH, MacEntee MI. Magnet-retained implant-supported overdentures: review and 1-year clinical report. J Can Dent Assoc 2010; 76(1):a52 (Review)

10. Chung C H, Choe H C, and Ha Kwak J: Degradation Phenomena of Magnetic Attachments Used Clinically in the Oral Environment, METALS AND MATERIALS International .2006, 12(4):pp. 357 364

11. Haffajee A D, Smith C, Torresyap G, Thompson M, Guerrero D and Socransky S S. Efficacy of manual and powered tooth brushes (II). Effect on microbiological parameters, J Clin Periodontol. 2001; 28: 947-954.

12. Hosoi T, Ohkubo C, Takada Y, Okuno O, Nakamura Y, Tanaka Y, Ishigami,Y T, Umekawa Y, Kikuchi A, Mizutani H:Foreign Dental Magnetic Foreign Dental Magnetic Attachments, The 7th International Conference on Magnetic Applications in Dentistry, March 3 - March 21,2008 .

13. Jena KA, Duggal R, and Barta P. Magnet as a dental material. An overview. Trends Biomater. Artif. Organs 2003, 16(2):73-80_

14. Kameda $\mathrm{T}$ and Ohkuma K: Electromagnetic Fields from Dental Devices and their Effects on Human Health, J Electr Electron Sys. 2014, 3:118
15. Kumar PS. Sex and the subgingival microbiome: do female sex steroids affect periodontal bacteria? Periodontol j 2000. 2013; 61(1):103-24.

16. Könönen $\mathrm{E}$ and Kumar $\mathrm{P}$ Bacteriology of periodontal diseases. In: Tangv YW, Sussman M, Liu D, Poxton I, Schwartzman J (eds) Molecular medical microbiology. 2015, Academic, Tokyo, pp 957-968

17. Kohno M, Yamazaki M, Kimura I, and Wad M. Effect of static magnetic fields on bacteria: Streptococcus mutans, Staphylococcus aureus, and Escherichia coli, Pathophysiology. 2000, 7(2): 143-148

18. Lemon J, Chambers M S, Wesley PJ. , and Martin, J. W. Technique for magnetic placement and orientation of a facial prosthesis. J. Prosthet. Dent 1996, 75: 50-2.

19. Madien, M.F. J.; Garman, R.J.; Curtis, M.A. ; Cillett, I.R.;Griffi G.S.; Sterne, J.A.C., Wilton, J.M.A.; and Johson. N.W.: Detection of high-risk groups and individuals for periodontal diseases: Laboratory markers based on the microbiological analysis of subgingival plaque, J. Clin. Periodontol; 1990, 17(1):1-13

20. Mcghee J R, Michalek SM and Cassel C H: Dental Microbiology Harper and Raw publisher, Philadelphia U.S.A $1^{\text {st }}$ edition1982, P654-662

21. Mombelli A, Minder, Ch. E.; Cusborti, and Lang NP. Reproducibility of microscopic and cultural data in repeated subgingival plaque samples J. ClinPeridontol. 1988, 16: 434

22. Newman M G, Socransky SS, Savitt ED, Propas D A and Grawford A: Studies of the microbiology of periodontitis, J. Periodontol. 1976, 47:373.

23. Morhart RE, Fitzgerald RJ.Nutritional determinants of the ecology of the oral flora. Dent Clin North Am, 1976 Jul; 20(3): 473-89.

24. Nirola A, Barta J, and Kaur J. Ascendancy of sex hormones on peridontium during reproductive life cycle of women. $\mathrm{J}$ Int Clin Dent Res Organ 2018; 10:3-11.

25. Omar AA, Newman HN, Bulman J, Osborn J. Darkground microscopy of subgingival plaque from the top to the bottom of the periodontal pocket. J Clin Periodontol. 1990, 17(6):364-70.

26. Parekh M,Pammi V,Vardhana B, Hiduja DM,Asnani M, and Ahmed A: Isolation and evaluation of microbial flora in patients with chronic periodontitis: a microbial study. journal of international oral health 2016;8(5):619-622. 
27. Portnoy, L. L.: Magnetic retention for overdentures and partial, CDS Review, Dec.1986

28. Raghavan R, Ramzi M, Ranjith Kumar P, Shajahan PA, Usman J, Balakrishnann S., Magnets in Complete denture, International journal of Oral Health Dentistry. 2015,1(3):133-137

29. Robbins J W .Success of overdentures and prevention of failure, .J A m Dent Ass, 1980, 100: 858

30. Savitt E D and Socransky S.S.: Distribution of certain subgingival microbial species in selected periodontal conditions, J. Periodontal. Res.1984, 19:111.

31. Shchipkova A Y, Nagaraja H N and Kumar P S. Subgingival microbial profiles of smokers with periodontitis: J.Dent. Res.2010,89(11); 1247-53.

32. Slots J. Subgingival micro - flora and periodontal disease, J. Clin. Periodontol. 1979, 6: 351.

33. Sullivan M, Casey DM, Alberico R, Litwin A and Schaaf NG. Hyperostosis in an orbital defect with craniofacial implants and open-field magnets: a clinical report. J Prosthet Dent. 2007; 97:196-199.
34. Tanner, A.C.R.; Socransky, S.S.; and Coodson, J, M.: Microbiota of periodontal pockets losing crestal alveolar bone, J. Periodont. Res., 19: 279, 1984

35. Tenford $\mathrm{T} \mathrm{S}$. Interaction mechanisms and biological effects of static magnetic fields US /japan seminar on electromagnetic fields effect June 28/July 1, 1994 Sapporo, Japan.

36. Thomas K. F.: Free standing magnetic retention for extra oral prosthesis with Osseo integrated implants. 1995. J. Prosthet. Dent, 73: 162.

37. White D, and Mayrand D. Association of oral Bacteroides with gingivitis and adult periodontitis. J Periodontal Res. 1981 May; 16(3):259-265.

38. Walker C B, Gordon J M, McQuilkin S J,. Niebloom, and, and T A, and Socransky S S, Tetracycline: Levels Achievable in Gingival Crevice Fluid and in Vitro Effect on Subgingival Organisms: Part II. Susceptibilities of Periodontal Bacteria. J periodontology 1981, 52(10) 609-612.

39. Williams, N. B. Microbial ecology of the oral cavity J Dent Res., 1963, 42: 509. 